\title{
Retraction Note: Landscape design of hill ecology and rural human settlement environment based on the analysis of geographic information system
}

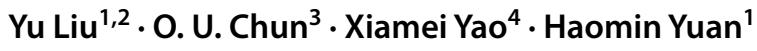

Published online: 13 December 2021

(c) Saudi Society for Geosciences 2021

Retraction Note: Arabian Journal of Geosciences (2021) 14: 1624

https://doi.org/10.1007/s12517-021-08022-1

The Editor-in-Chief and the Publisher have retracted this article because the content of this article is nonsensical. The peer review process was not carried out in accordance with the Publisher's peer review policy. Author Ou Chun disagrees with this retraction. Authors Yu Liu, Xiamei Yao and Haomin Yuan have not responded to correspondence regarding this retraction.

The original article can be found online at https://doi.org/10.1007/ s12517-021-08022-1.

O. U. Chun

ouchun2021@163.com

Yu Liu

718111060@qq.com

Xiamei Yao

yaoxiamei1209@126.com

Haomin Yuan

1135744969@qq.com

1 School of Architecture and Engineering, Suqian College, Suqian 223800, Jiangsu, China

2 School of Biological and Environmental, Nanjing Forestry University, Nanjing 210095, Jiangsu, China

3 School of Biology and Food Engineering, Fuyang Normal University, Fuyang 236037, Anhui, China

4 School of Architecture and Urban Planning, Anhui Jianzhu University, Hefei 230022, Anhui, China 